\title{
Design of New Test Function Model Based on Multi-objective Optimization Method
}

\author{
SHANG Zhaoxia ${ }^{1, *}$, ZHANG Rui $^{2}$, WANG Jiankang $^{1}$, and ZHANG Duo ${ }^{1}$ \\ ${ }^{1}$ Shandong Product Quality Inspection Research Institute, Jinan250102, Shandong, China. \\ ${ }^{2}$ Shandong Institute of medicine and health information, Jinan250062, Shandong, China.
}

\begin{abstract}
Space partitioning method, as a new algorism, has been applied to planning and decision-making of investment portfolio more and more often. But currently there are so few testing function for this algorism, which has greatly restrained its further development and application. An innovative test function model is designed in this paper and is used to test the algorism. It is proved that for evaluation of space partitioning method in certain applications, this test function has fairly obvious advantage.
\end{abstract}

Keywords: Multiple-objective optimization, test function, investment portfolio, model design, space partitioning method

\section{Introduction}

Test function is an important tool for algorism evaluation. Generally it evaluates an algorism's performance from 3 aspects - time complexity, distribution and convergence. Whitley ${ }^{[1]}$ proposed a guideline for test function design in 1996. $\mathrm{Deb}^{[2]}$ divided the design method of test function into three types. In addition, the test function proposed by literature ${ }^{[3]}$ in 1999 is widely accepted. A test function of alterable decision variables dimension was proposed in the literature ${ }^{[4]}$. An index for distribution assessment proposed by Schott, et $\mathrm{al}^{[5]}$ is the most popular parameter now. In 2010, Ishibuchi et $\mathrm{al}^{[6]}$ proposed to evaluate the fitness of the individual using different Scalarizing Functions in MOEA/D at the same time. In recent years, new test functions are proposed one after another. Some of them are already well-known such as the test method based on non-dominated sorting proposed by Srinivas $^{[7]}$, the test method based on genetic algorithm proposed by $\operatorname{Deb}^{[8]}$, and the scalable multi-objective test toolkit proposed by Huband $^{[9]}$.

\footnotetext{
* Corresponding author: gogogo255@163.com
} 
The test function must be closely related to the problem solved by the algorism, in other words, individualized test function shall be adopted according to characteristics of the problem to make it worthy. A large portion of current functions are universal functions based on historical data and complex solution; there are quite few specific test functions facing certain type of problems. In view of the above limitation, a new test function for space partitioning application in investment portfolio as well as the performance measurement index is designed in this paper. Considering the requirement of portfolio on risk scattering, the design makes a point of test stability and distribution uniformity; considering the investor may want to control and intervene in the decision-making process, this test function is designed to be visualized and controllable.

Since the test function is designed for space partitioning method, distribution uniformity has to be the most important evaluation index. The solution set which distributes evenly can not only provide better options to the decision-maker, but also affect search capability of the algorism to a large degree ${ }^{[10]}$. There are multiple possible reasons for nonuniform distribution of population. For example, the target function is nonlinear or has multiple variables; parameters of different target functions are interrelated; nonlinear mapping exists between decision variables and parameters; the Pareto frontier is inconsecutive [11], etc. Therefore the test function proposed in this paper is designed not only to avoid that the population fall in the optimal zone of a single target function, but also to reflect restrictive relation between target function and solution through assessing the distribution uniformity. Most algorism just considers the impacts of the entirety on the individuals and does not consider the impacts between individuals. But in some practical cases, the affect is although small, but can't be ignored. Therefore, this article fully considers the influence between individuals, and also screens the running solution and performs maintenance on the external population by using test function.

The algorism proposed in this paper showed great superiority in the application field which have high request for dispensability. For example, in specific application of portfolio decision-making and planning, the investor values risk diversification of portfolio, avoid the combination plan is too single and close, and also avoid deviating from the actual. It is the major need for avoiding the interest rate risk. Correspondingly, the stability and distribution evenness were highly stressed during the designing of the test algorism.

\section{Experimental}

Firstly define the test function as: 


$$
\mathrm{TES}_{\mathrm{i}}=\alpha^{2}+\beta \frac{K}{k}
$$

$\mathrm{i}$ is the individual code; $\alpha$ is the number of individuals which fall in the grid where i stays; $\beta$ is the number of individuals which fall in adjacent grids; $\mathrm{K}$ is the number of all possible grids in adjacent region; $\mathrm{k}$ is the number of grids in valid adjacent regions. Higher TES ${ }_{i}$ represents smaller fitness of individuals. Reason is that in a certain region, more densely the individuals are distributed, less contributions they make to the population. Accordingly these individuals have lower chance to be chosen. The evaluation of an algorism's performance shall also include deviation of its result to the actual result. Therefore the fuzzy expert evaluation system shall be set. First step is to integrate opinions of the experts and regulate them as Pro ${ }_{i}$ value, which is called as expert evaluation index. Then the index can be screened and tested as a decision factor after TES ${ }_{\mathrm{i}}$. The secondary fuzzy expression of the test function is:

TES $_{i}=\alpha^{2}+\beta \frac{K}{k}+\operatorname{Pro}_{i}$

Then grant weight to two evaluation aspects; the secondary fuzzy expression changes to:

$\mathrm{TES}_{\mathrm{i}}=\mathrm{W}_{\mathrm{T}}\left(\alpha^{2}+\beta \frac{K}{k}\right)+\mathrm{W}_{\mathrm{P}} \mathrm{Pro}_{\mathrm{i}}$
It shall satisfy:

$\mathrm{W}_{\mathrm{T}}+\mathrm{W}_{\mathrm{P}}=1$

$\mathrm{W}_{\mathrm{T}}$ is objective weight; $\mathrm{W}_{\mathrm{P}}$ is subjective weight. The calculation procedure without expert evaluation index shows as following:

Step 1. Define scale of the population.

Step 2. Get TES $\mathrm{i}_{\mathrm{i}}$ value of the solutions sets.

Step 3. Re-rank --- according to TES ${ }_{\mathrm{i}}$.

Step 4. Compare the results of two ranking and evaluate the algorism.

Step 5. Move out or move in individuals from external population according to the result of step 4

Step 6. Maintain the population according to Pro $_{i}$.

\section{Simulation}

The original test results generated by using method of literature ${ }^{[12]}$ are shown in table 1. Then re-rank these results by $\mathrm{TES}_{\mathrm{i}}$ value using the method proposed in this paper. The new results are shown in table 2.

\begin{tabular}{ccccc}
\multicolumn{5}{c}{ Table 1 Original Test Results } \\
\hline No. & 1 & 2 & 3 & 4 \\
\hline Indivi & $18,(4,2-1$, & $5,(4,2$, & $4,(5,2-1$, & $1,(5,2-2$, \\
dual & $2.000000)$ & $2.000000)$ & $2.400000)$ & $2.400000)$ \\
\hline TES & 7 & 7 & 7 & 7 \\
\hline No. & 5 & 6 & 7 & 8 \\
\hline Indivi & $6,(3,1$, & $15,(6,2$, & $22,(3,4$, & $25,(8,1$, \\
dual & $2.800000)$ & $2.800000)$ & $2.800000)$ & $3.000000)$ \\
\hline TES & 6.3 & 6 & 6.3 & 6.3 \\
\hline
\end{tabular}




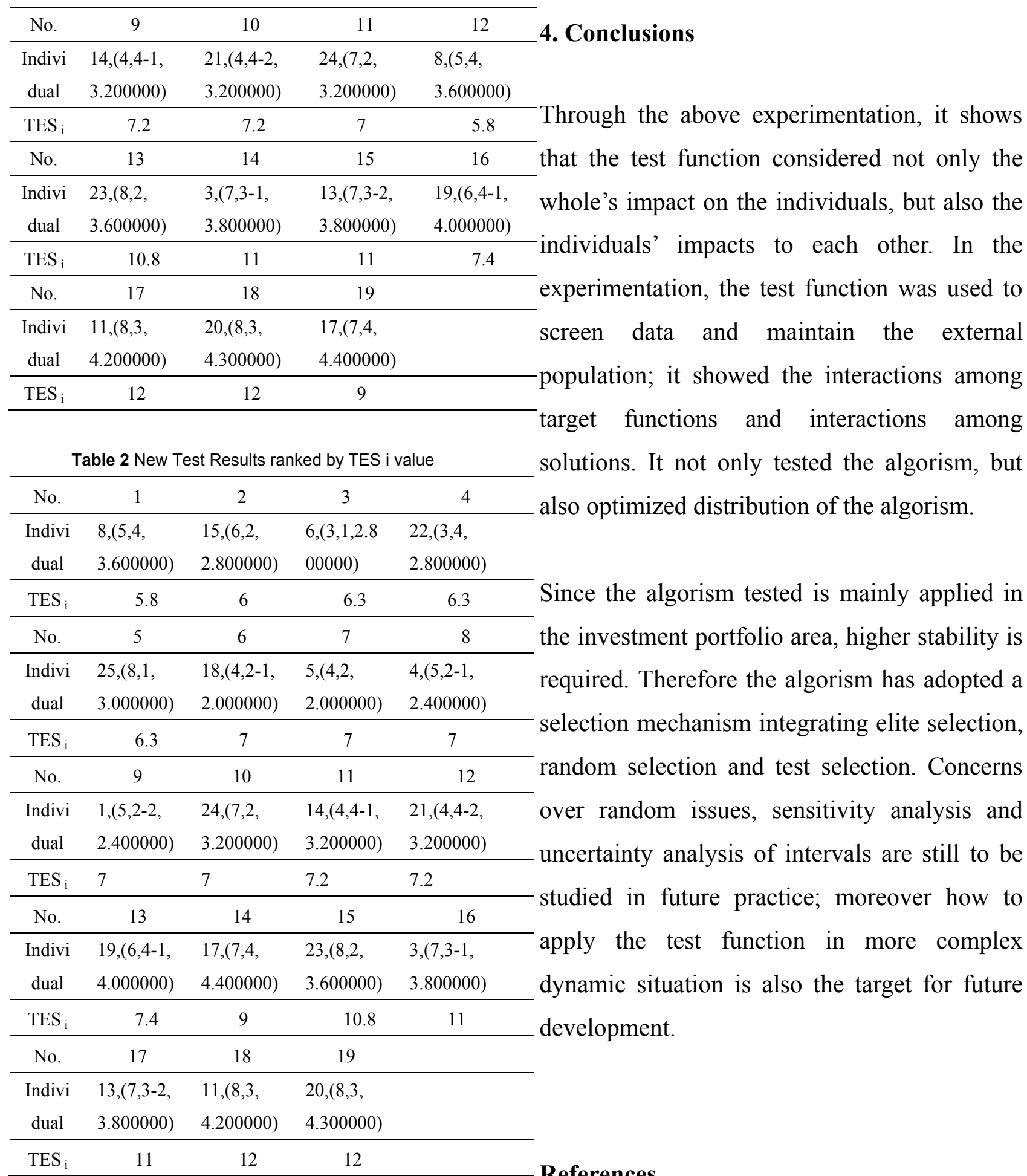

Through comparison and analysis of the above two ranking results, the algorism can be evaluated. It shows that the algorism has comparatively low congestion and good distribution.

1. Whitley D, Rana S, Dzubera J, et al. Evaluating evolutionary algorithms.Artificial Intelligence, 1996, 85(1-2): 245-276

2. Abraham A, Jain L, Goldberg R. Evolutionary Multi-objective Optimization: Theoretical Advances and Applications. London: Springer, 2005 
3. Van Veldhuizen David A, Lamont G B. Multiobjective evolutionary algorithm test suites. In: Carroll J, et al. Editors, Proceedings of the 1999 ACM Symposium on Applied Computing, 1999: 351-357

4. Fonseca Carlos M, Peter J Fleming. Multiobjective genetic algorithms made easy: selection, s haring, and mating restriction. In: Proceedings of the First In ternational Conference on Genetic Algorithms in Engineering Systems: Innovations and Applications, 1995: 42-52

5. SCHOTT J R. Fault tolerant design using single and multicriteria genetic algorithm optimization. Cambridge: Massachusetts Institute of Technology, 1995

6. Ishibuchi, Hisao, et al. "Simultaneous Use of Different Scalarizing Functions in MOEA/D." Genetic and Evolutionary Computation Conference, GECCO 2010, Proceedings, Portland, Oregon, Usa, July 2010:519-526.

7. Srinivas N, Deb K. Multiobjective Optimization Using Nondominated Sorting in Genetic Algorithms. Evolutionary Computation, 1995, 2 (3): 221-248

8. Deb K, Prat a PA, Agarw al S, et al. A Fast and Elitist Multiobjective Genetic Algorithm: NSGA-II. IEEE Transactions on Evolutionary Computation, 2002, 6: 182-197

9. Huband S, Barone L, While L, et al. A Scalable Multi-objective Test ProblemToolkit. Proceedings of the Evolutionary Multi-criterion Optimization'05, Berlin, Germany: Springer, 2005: 280-295

10. Laumanns M, Thiele L, Deb k, et al. Combining convergence and diversity in evolutionary multiobjective optimization. Evolutionary Computation, 2002, 10(3): 263-282

11. Cheng P, Zhang Zili. Design and analysis of test problems of multi objective evolutionary algorithm. computer project, 2009, 35(14): 238-240(in Chinese)

12. Shang Z X, Liu H. A Multiple-Objective Algorism
Design and Strategy Study Based on Target Space Partitioning. Advanced Materials Research, 2011, 1228: 219-220(in Chinese) 